\title{
THE DIRECT HEALTH COSTS OF INFLAMMATORY POLYARTHRITIS TEN YEARS AFTER DISEASE-ONSET: RESULTS FROM THE NORFOLK ARTHRITIS REGISTER
}

Elena Nikiphorou ${ }^{1,2}$, Charlotte Davies ${ }^{3}$, Miranda Mugford ${ }^{3}$, Nicola Cooper ${ }^{4}$, AlanBrooksby $^{5}$, Diane K Bunn ${ }^{3}$, AdamYoung ${ }^{2}$, Suzanne M.M. Verstappen ${ }^{6}$, Deborah P.M Symmons ${ }^{6,7}$, Alex J. MacGregor ${ }^{3}$

1. Department of Applied Health Research, University College London, London

2. Centre for Lifespan \& Chronic Illness Research, University of Hertfordshire, Hatfield

3. Norwich Medical School, University of East Anglia, Norwich

4. Department of Epidemiology and Public Health, University of Leicester, Leicester

5. Clinical Effectiveness and Audit Department, Norfolk \& Norwich University Hospital, Norwich

6. Arthritis Research UK Centre for Epidemiology, University of Manchester, Manchester

7. NIHR Manchester Musculoskeletal Biomedical Research Unit

Corresponding Author:

Professor Alex MacGregor

Faculty of Medicine and Health Sciences

University of East Anglia

Norwich Research Park

Norwich

NR4 7TJ United Kingdom

Email: a.macgregor@uea.ac.uk

Key indexing terms: Inflammatory polyarthritis, rheumatoid arthritis, health service provision, direct health costs.

Disclosure statement: The authors have declared no conflicts of interest.

Research funded by: Action Arthritis. The Norfolk Arthritis Register is funded by Arthritis Research UK (Reference 20380). 


\section{Abstract}

Objectives. To explore the change in direct medical costs associated with inflammatory polyarthritis (IP) 10 to 15 years after its onset.

Methods. Patients from the Norfolk Arthritis Register (NOAR) who had previously participated in a health economic study in 1999 were traced ten years later and invited to participate in a further prospective questionnaire-based study. The study was designed to capture direct medical costs and changes in health status over a 6-month period, using previously validated questionnaires as the primary source of data.

Results. A representative sample of 101 patients with IP from the 1999 cohort provided complete data over the 6-month period. The mean disease duration was 14 years (SD 2.1, median 13.6, IQR 12.6-15.4). The mean direct medical cost per patient over the 6month period was: $£ 1496$ for IP (inflated for 2013 prices). This compared with $£ 582$ (95\% CI £355-£964) inflated to 2013 prices per patient with IP ten years earlier in their disease. The increased cost was largely associated with use of biologics in the RA subgroup of patients (51\% of total costs incurred). Other direct cost components included primary care costs $11 \%$; hospital outpatient $19 \%$; day care $12 \%$; inpatient stay $4 \%$.

Conclusions. The direct health care costs associated with IP have more than doubled with increasing disease duration, largely as a result of the use of biologics. The results showed a shift in the direct health costs from inpatient to outpatient service use.

\section{Introduction}


Inflammatory arthritis (IP), of which rheumatoid arthritis (RA) is a subset, is a chronic and disabling condition with a lifelong course and considerable economic impact both on the affected individual and on society.[1] In 1992, the annual costs of RA in England were estimated to be $£ 1.26$ billion [2] with the direct costs (the use of primary and secondary care services and drug costs) accounting for $48 \%$ of the total, the remainder reflecting indirect health costs. Almost 20 years later, the estimated cost of RA in England has risen to approximately $£ 8$ billion a year (DH drug costs 2011).[3]

Establishing the true cost associated with IP is complex because costs change over the time course of the disease with age, disease progression and changing treatment strategies. Secular changes in disease severity, the earlier introduction of disease modifying agents and the use of targeted management regimes together with the increasing availability of biologic agents, are all likely to have complex and competing effects on long term costs.[4]

In 1999, as part of a wider assessment of the costs of new-onset IP, we examined the costs of disease using a cohort of 133 consecutive patients from the Norfolk Arthritis Register (NOAR), recruited between 1994 and 1999.[5] A total 50\% subsequently developed RA. An analysis of the data obtained using a validated resource use and expenditure questionnaire $[5,6]$ revealed that, in the first five years after disease onset, direct health costs represented $14 \%$ of the total, the main component being in-patient and day care stays (33\%). This was followed by prescribed medication, which accounted for $30 \%$ of the total health service costs.

In this follow up cross-sectional study we re-evaluate the cost of the disease after a further 10 years in a sample taken from the same cohort of patients. This community based longitudinal cohort design provides a unique opportunity to observe the changing costs of treatment associated with IP and its progression against a changing therapeutic backdrop.

\section{Methods}




\section{Study subjects}

NOAR is a long-term prospective cohort of over 4,000 patients with new onset IP (>2 swollen joints lasting $>4$ weeks) identified from primary care and hospital sources within the boundaries of the former Norwich Health Authority UK, starting in 1990 and currently on-going. [7,8]

In 1999 a health economic evaluation was conducted prospectively over a 6-month period among 133 patients with IP in the community, using self-completion postal questionnaires, designed and validated for the study.[5] These questionnaires (available as supplementary material) included detailed sections on primary care and hospital visits (to see doctors, nurses or other health care professionals), prescribed medications and over-the-counter drugs. An extra question was added to the original questionnaires under the medications section, to capture the use of biologic DMARDs in the current study. Any reporting of biologic DMARD use was checked against the patients' paper records to ensure accurate recording of information. Questions on medications included the type of drugs used, dosage, number of prescriptions and duration of treatment, enabling the calculation of costs using standard UK prices (see below) at individual patient level. A second cross-sectional study, which also took place in 1999, recruited 218 NOAR participants. Both studies in 1999 were designed to estimate the health service (direct health costs), non-health service (e.g. over the counter medications) and total costs of early IP.[5] The subjects recruited were consecutive patients entering NOAR between 1994-1999. When interviewed by the NOAR research nurses at the time of recruitment, 267 patients had given permission for further contact by the register and could be located in 2009. The remaining patients had either died, declined NOAR in the intervening period, were untraceable or had pre-arranged agreement with the study team in 1999 not to be contacted again.

\section{6 month prospective evaluation}

All 267 eligible study subjects in 2009 were contacted by letter (with a reminder after two weeks if they failed to respond) inviting them to participate in a 6 month prospective healthcare evaluation survey (Reference 2003RH06 119-09-03). A total of 141 consented to participate. Resource use and expenditure data were gathered at baseline (namely, at the start of this study), 3 and 6 months by self-completion of the same postal questionnaire used in 1999. 
As in our previous study, [5] study participants were provided with a memory aid in the form of a diary to record their resource use and expenditure over the 3-month follow-up intervals. Completion of the diary was optional and intended for the participants' personal use only.

The NOAR database holds information on socio-demographic data including age, sex, smoking status, social class and ethnic group; social class was assigned using the Registrar General's system Office of Population Censuses and Surveys, 1991,[9] based on the occupation of the individual. Other clinical, laboratory and radiographic data recorded in the NOAR database include the 28-joint disease activity score (DAS 28), Health Assessment Questionnaire (HAQ), C-reactive protein (CRP), rheumatoid factor and/or cyclic citrullinated peptide antibody (anti-CCP) and presence of erosions on Xrays. Details on disease-modifying anti-rheumatic drug (DMARD) use were documented at each patient review.

\section{Analytical approach (cost calculations)}

Unit costs were applied to the resource-use data using data from the Personal Social Services Research Unit 2009 (PSSRU) and for medications, the British National Formulary (BNF) 2009 was used.[10] Medications relating to IP treatment, including analgesics, DMARDs and bone-strengthening treatments were costed individually using the BNF and based on the number of prescriptions, assuming that each prescription was for one month (from the data available). With regards to the biologics, only etanercept and adalimumab were prescribed in this study population. The BNF (2009) reports the price of both anti-TNFs as $£ 89.38$ per 25 -mg prefilled syringe for etanercept (standard dose of $25 \mathrm{mg}$ twice weekly or $50 \mathrm{mg}$ once weekly) and $£ 357.50$ per 40 -mg prefilled syringe for adalimumab (standard dose $40 \mathrm{mg}$ on alternate weeks).

The direct health costs per participant over 6 months was estimated following the same methodology as reported by Cooper et al.,[5] in the earlier study. We combined resource use data with the unit costs using the following general costing formula:[5]

$$
\text { Total cost }=\sum_{i=1}^{n} \sum_{j=1}^{m}(\text { frequency })_{i j} \times(\text { unit cost })_{j}
$$


where $i$ is the $i$ th individual $(i=1 \ldots \ldots \ldots n)$ and $j$ is the $j$ th service received $(j=1 \ldots \ldots . . m)$ (i.e., inpatient and outpatient care, and different second line drugs).

All costs were inflated to 2013 prices (Bank of England inflation calculator).[11] A table of unit costs is available online, where all costs are expressed in 2013 UK sterling.

Separate cost categories were then defined, including: hospital, community and treatment costs. Health care costs are typically right skewed[12] due to the small number of patients reporting high treatment costs compared with the population as a whole. For interpretation of our results, we report the following descriptive statistics for each variable:

1. Number and proportion (\%) of participants using specific services.

2. Mean, standard deviation and the $95 \%$ confidence intervals for costs calculated on all resource use.

The individual costs per patient were calculated for the 6 month period, using STATA 11 and Microsoft Excel.

\section{Results}

\section{Patient sample description}

Of the 141 responders who consented to participate, $101(72 \%)$ returned complete data at baseline, 3 and 6 months. Their baseline characteristics are shown in Table 1. Their mean age was 49.8 years (SD 12.0); 63\% were female and mean disease duration was 14 years (SD 2.1, median 13.6, IQR 12.6-15.4). The baseline characteristics in the present study were broadly similar to the baseline characteristics of the subjects originally included in the 1999 study, the only difference related to their longer disease duration. The patients in the current study had comparable age with the 1999 cohort (mean 50 years vs 52 years respectively) similar disease duration at entry (47 vs 51 months), gender (female 64\% vs 62\%) and HAQ at entry (median 0.63 vs 0.75 ). Compared to the 1999 study, as would be expected with longer follow up, a greater proportion of patients (63\% compared to 50\%) had been classified by ACR criteria[13] as having RA. Biologic drugs were used by 14 patients (14\%) during the 6-month study period. As expected, those who developed established RA during follow up showed higher DAS28, CRP and HAQ at baseline than the group overall. 


\section{Costs of disease}

The total direct cost incurred by all IP ( $\mathrm{n}=101)$ patients was $£ 128,903$ over the 6 month study period, with a mean cost of $£ 1,276$ (95\% CI £856-£1696) (£1,493 inflated for 2013 prices) per patient and a Total Annual Direct cost (TAD) of $£ 2,556.00$ ( $£ 2,991$, inflated for 2013 prices). Medications and biologic drugs accounted for the largest proportion of the total health care cost (54\%), followed by hospital outpatient visits and day care admission (31\%). Inpatient hospital stay for orthopaedic intervention was required for two patients in the 6-month follow-up interval. Inpatient admissions accounted for $4 \%$ of the total cost in this sample. Outpatient hospital visits (including both consultations with doctors and nurses) accounted for $19 \%$ of the cost for IP and use of day unit facilities accounted for $12 \%$. Community visits, including visits to the General Practitioner (GP), GP nurse and community physiotherapy, and occupational therapy accounted for $11 \%$ of the total cost of IP.

In those who developed RA $(n=64)$ the mean total cost per patient over the 6 month period was $£ 1,715$ (95\% CI $£ 1,105-£ 2,326$ ), TAD cost $£ 3,430$ ( 22,007 and $£ 4,014$ respectively inflated for 2013 prices). Use of biologic drugs accounted for $51 \%$ of the total direct costs, despite only $14 \%$ of the cohort having been prescribed these drugs. Other IP-related medications accounted for an additional $6 \%$ of the cost.

The costs of all IP patients and also the disaggregated costs for the RA group over the 6 month study period are reported in Table 2 displaying the $50^{\text {th }}$ percentile (median), $75^{\text {th }}$ percentile and maximum values to allow for a clear representation of the distribution of the data.

\section{Discussion}

The previous literature on the economic impact of IP and RA has focussed on the shortterm direct medical costs. This is the first study to capture the associated long-term direct medical costs, in an era where emphasis is placed on the use of earlier and more intensive treatments. There has been a shift from a limited number of drugs with slow acting and/or marginal effects to use of more potent drugs such as the biologics, when first line synthetic DMARDs fail to control disease. The clinical effectiveness and 
benefits of biologic agents on both short-term and longer-term outcomes of disease is now well-established. However, they do represent the most expensive type of medication for IP and this restricts their use in the UK since their availability in 2002. This study has shown that up to 15 years after the onset of IP, biologic agents accounted for over $50 \%$ of the total cost associated with disease.

However, like-for-like comparison with our earlier 1999 study for individual resource use is not possible because of methodological differences, individual cost components being grouped differently and the focus of the study being on both direct and indirect costs. The 1999 study was not designed with long term follow up of health economic outcomes in mind, and a group of patients were not consented for further questionnaire contact. While the present and original cohorts were broadly similar in their baseline characteristics, the possibility of selective difference influencing the comparison cannot be excluded fully. Nevertheless, we believe valid comparisons of broad trends for the most notable differences over time are possible. In the 1999 study a greater proportion of the total 6-month health-service costs $(42 \%)$ were attributable to a minority of individuals who incurred an in-patient stay or day-care visit $(6 \%)$. These 'high cost' individuals represented $10 \%$ of the cohort and incurred $50 \%$ of the total 6 month costs (both health and non-health service).

In the current study, outpatient visits and day care admission accounted for just under one third of the cost (31\%), whereas inpatient admission was only reflected by $4 \%$ of the cost over the 6 month period. The in-patient admissions were for orthopaedicrelated interventions. The observed shift from inpatient to outpatient care could be due to better management and more intensive treatment of patients in the outpatient setting and could relate to the use of biologic agents. However, we can only speculate on this observation which could also simply be a reflection of the changing approach to delivering care to patients with IP in the UK.

The mean direct (health service) medical cost per patient over the 6-month period in this study was $£ 1,493$ for all IP (inflated for 2013 prices). This compared with $£ 582$ (95\% CI £355-£964) inflated to 2013 prices (£385; 95\% CI £235-£638 in 1999 prices) per patient with IP ten years earlier in their disease.[5] There has, therefore, been an obvious increase in costs, with the use of biologics being a major contributor. 
Whilst direct comparisons between studies based on differing healthcare systems using different economic evaluations is not possible, it is of interest that our figures are comparable to those reported in studies from the USA, Canada and Europe, accounting for inflation. [14,15] In a prospective longitudinal study of costs incurred by RA patients in Saskatoon and Montreal [14], annual direct costs increased by almost $20 \%$ in the late 1980s compared to the early 1990s. Institutional stays and medications made up at least $80 \%$ of total direct costs, the former being more frequent in the older and more disabled as one would expect. In this study that was published in 1997, the authors concluded that measures to reduce long-term disability by using earlier and more aggressive intervention have the potential to produce considerable cost savings. However, it was unknown then which strategies would have the greatest effect on outcome and the optimal allocation of resources. Direct comparison of the results with other studies can be challenging and may be inappropriate, due to differences in health systems, population characteristics e.g. age, gender, disease severity and duration[1] as well as methodological differences e.g. different cost components, unit costs, etc. Therefore, generalisation of the results to a wider geographical area and other healthcare systems is not possible and is an unavoidable limitation of the study.

The study is limited by the fact that information on the indirect health care costs (such as productivity losses to the individual, family, employer and society as a result of illness) was not sufficiently and robustly collected, and we anticipate that our results will have underestimated the full societal cost of the disease. Details on investigational costs e.g. for imaging and blood tests were not included, which could result in underestimation of costs. However, our aim was to provide a description of the direct medical costs in this cohort of patients with a view to assessing the changing cost of disease and healthcare utilization group over time. By adopting similar methodology to our earlier study we provide an overall assessment of their inpatient and outpatient care. Finally, the use of self-reported questionnaires to collect information on resource and drug use and costs is a limitation of the study. However, we tried to minimise the risk of patient recall bias, by providing study participants with a memory aid in the form of a diary in which to record their resource use and expenditure over the two 3-month follow-up intervals. 
One of the key issues surrounding the use of biologic agents in IP is whether the long term cost of the disease will be reduced by the introduction of more effective treatments earlier in the disease. Comparative data will be needed from later cohorts to provide insight into this. However the present study has shown that biologic use among this cohort currently dominates the direct cost of the long term management of IP when compared to all other aspects of direct medical care. 
Costs of inflammatory polyarthritis

\section{References}

1. Cooper NJ. Economic burden of rheumatoid arthritis: a systematic review. Rheumatology (Oxford) 2000;39:28-33.

2. McIntosh E. The cost of rheumatoid arthritis. Br J Rheumatol 1996;35:781-90.

3. www.nras.org.uk National Rheumatoid Arthritis Society. The economic burden of rheumatoid arthritis. March 2010 Available from:

http://www.nras.org.uk/publications/the-economic-burden-of-rheumatoid-arthritis

4. Symmons DP, Silman AJ. The Norfolk Arthritis Register (NOAR). Clin Exp Rheumatol Suppl 2003;21 Suppl 31:S94-9.

5. Cooper NJ, Mugford M, Symmons DP, Barrett EM, Scott DG. Total costs and predictors of costs in individuals with early inflammatory polyarthritis: a communitybased prospective study. Rheumatology (Oxford) 2002;41:767-74.

6. Cooper NJ, Mugford M, Symmons DP, Barrett EM, Scott DG. Development of resource-use and expenditure questionnaires for use in rheumatology research. $\mathbf{J}$ Rheumatol 2003;30:2485-91.

7. Symmons DPM, Barrett EM, Bankhead CR, Scott DGI, Silman AJ. The incidence of rheumatoid arthritis in the United Kingdom: results from the Norfolk Arthritis Register. Br J Rheumatol 1994;33:735-9.

8. Symmons D, Turner G, Webb R, Asten P, Barrett E, Lunt M, Scott D, Silman A. The prevalence of rheumatoid arthritis in the United Kingdom: new estimates for a new century. Rheumatology (Oxford) 2002;41:793-800.

9. Office of Population Censuses and Surveys. Standard occupational classification, Vol. 3. Social classifications and coding methodology. London HMSO; 1991; (ISBN) 10:011691338X 
Costs of inflammatory polyarthritis

10. British Medical Association and the Royal Pharmaceutical Society of Great Britain. British National Formulary 58th ed. 2009: BMJ Publishing Group no. (ISBN) 13:9780853698487

11. Bank of England.co.uk [homepage on the Internet]. London: Bank of England Inflation Calculator. [updated 2014 July 9] Available from: http://www.bankofengland.co.uk/education/Pages/resources/inflationtools/calculator/ind ex1.aspx

12. Briggs $\mathrm{AH}$, and Gray AM. Handling uncertainty when performing economic evaluation of healthcare interventions. Health Technol Assess 1999;3:1-134.

13. Arnett FC, Edworthy SM, Bloch DA, McShane DJ, Fries JF, Cooper NS, et al. The American Rheumatism Association 1987 revised criteria for the classification of rheumatoid arthritis. Arthritis Rheum 1987;31:315-24.

14. Clarke AE, Zowall H, Levinton C, Assimakopoulos H, Sibley JT, Haga M, et al. Direct and indirect medical costs incurred by Canadian patients with rheumatoid arthritis: A 12 year study. J Rheumatol 1997;24:1051-60.

15. Jonsson B, Rehnberg C, Borgquist L, Larsson SE, et al. Locomotion status and costs in destructive rheumatoid arthritis - a comprehensive study of 82 patients from a population of 13,000. Acta Orthop Scand 1992;63:207-12. 
Costs of inflammatory polyarthritis

Table 1. Patient characteristics by RA status at the time of recruitment into the study.

\begin{tabular}{|l|c|c|}
\hline \hline & $\mathbf{I P}$ & $\mathbf{R A}$ group* \\
& $\mathbf{N}=\mathbf{1 0 1}$ & $\mathbf{6 4}$ \\
\hline \hline Age at onset, years (mean. s.d) & $49.8(12.0)$ & $51.1(11.5)$ \\
\hline \hline Disease duration, years (mean, s.d.) & $14(2.1)$ & $14.4(2.2)$ \\
\hline \hline Gender, female & $64(63 \%)$ & $49(77 \%)$ \\
\hline \hline HAQ score (median, range) & $0.63(0.13-1.19)$ & $0.88(0.38-1.38)$ \\
\hline \hline DAS28 crp(3) (mean, s.d) & $3.6(1.4)$ & $4.2(1.1)$ \\
\hline \hline CRP, mg/L(median, range) & $7(0-15)$ & $11(3-23)$ \\
\hline \hline Swollen joint count, 28 joints (median, range) & $3(1-8)$ & $5.5(2-11)$ \\
\hline \hline Tender joint count, 28 joints (median, range) & $4(1-6)$ & $5(2-9)$ \\
\hline \hline RF, positive & $32(34 \%)$ & $28(45 \%)$ \\
\hline \hline Anti-CCP, positive & $27(31 \%)$ & $25(44 \%)$ \\
\hline \hline Erosions, yes & $10(51 \%)$ & $9(69 \%)$ \\
\hline & & $24(38 \%)$ \\
\hline
\end{tabular}

$N=$ number of patients with available data. Mean, standard deviation (s.d.) reported for all normallydistributed variables; median and inter-quartile range (IQR) reported for non-normally distributed variables. Percentages of total number shown in parentheses.

*classified by ACR criteria applied at 5 years after disease onset 
Costs of inflammatory polyarthritis

Table 2. Disaggregated costs for IP and the RA sub-group.

\begin{tabular}{|c|c|c|c|c|c|c|c|c|}
\hline & \multicolumn{4}{|c|}{ IP (n=101) } & \multicolumn{4}{|c|}{ RA group (n=64) } \\
\hline & \multicolumn{4}{|c|}{ Cost (f) } & \multicolumn{4}{|c|}{ Cost (f) } \\
\hline Resource use & $\begin{array}{c}N \text { using } \\
(\%)\end{array}$ & $\begin{array}{c}50^{\text {th }} \mathrm{PL}, 75^{\text {th }} \mathrm{PL}, \\
\max \end{array}$ & s.d. & $95 \% \mathrm{Cl}$ & $\begin{array}{c}N \text { using } \\
(\%)\end{array}$ & $\begin{array}{c}50^{\text {th }} P L, 75^{\text {th }} \\
\text { PL, max }\end{array}$ & s.d. & $95 \% \mathrm{Cl}$ \\
\hline GP nurse visits & $55(54)$ & $33,169,950$ & 193.36 & $142.09-246.71$ & $43(67)$ & $84,203,950$ & 189.98 & $139.65-256.61$ \\
\hline GP nurse calls & $14(14)$ & $0,0,77$ & 24.19 & $20.26-44.12$ & $10(16)$ & $0,0,77$ & 22.75 & $18.49-51.03$ \\
\hline NP visits & $40(40)$ & $0,50,754$ & 170.19 & $110.39-219.26$ & $34(53)$ & $50,100.65,754$ & 179.45 & $98.74-223.96$ \\
\hline NP calls & $16(16)$ & $0,0,38$ & 11.02 & $16.65-28.40$ & $13(20)$ & $0,0,38$ & 10.70 & $16.30-29.24$ \\
\hline GP visit & $47(46)$ & $0,72,544$ & 96.57 & $36.28-55.01$ & $33(52)$ & $36,72,544$ & 16.03 & $56.40-121.71$ \\
\hline GP call & $15(15)$ & $0,0,400$ & 96.28 & $31.19-137.82$ & $11(17)$ & $0,0,400$ & 109.56 & $5.24-152.45$ \\
\hline Hospital doctor visits & $56(55)$ & $143,294,1917$ & 294.94 & $250.20-291.12$ & $41(64)$ & $147,294,884$ & 209.82 & $232.32-364.78$ \\
\hline Hospital doctor calls & $13(13)$ & $0,0,333$ & 87.63 & $18.89-124.80$ & $11(17)$ & $0,0,333$ & 93.82 & $3.68-129.74$ \\
\hline Day care stay & $9(9)$ & $0,0,5227$ & 1627.51 & $740.56-3242.36$ & $7(11)$ & $0,0,5227$ & 1693.48 & 780.73-3913.14 \\
\hline Inpatient stay & $2(2)$ & $0,0,3073$ & 0 & $3073.63-3073.63$ & $2(3)$ & $0,0,3073.63$ & 0 & $3073.63-3073.63$ \\
\hline Physiotherapy & $16(16)$ & $0,0,252$ & 55.90 & $66.99-126.57$ & $10(16)$ & $0,0,126$ & 29.97 & $63.88-106.76$ \\
\hline Occupational Therapy & $13(13)$ & $0,0,126$ & 35.05 & $51.74-94.11$ & $11(17)$ & $0,0,126$ & 37.63 & $46.57-97.11$ \\
\hline Total medication use & $81(80)$ & $30,845,103$ & 190.11 & 87.89-171.96 & $56(88)$ & $39,121,845$ & 191.96 & $83.85-163.25$ \\
\hline Total cost biologics & $14(14)$ & $0,0,5021$ & 0 & $5021.42-5021.42$ & $13(20)$ & $0,0,5021$ & 0 & $5021.42-5021.42$ \\
\hline
\end{tabular}

$G P=$ General Practitioner NP=Nurse practitioner. $50^{\text {th }} P L=50$ th percentile (median); $75^{\text {th }} P L=75^{\text {th }}$ percentile; max=maximum value. All costs are reported in $£$ sterling and for the price year $2013 . \%$ indicates the percentage usage of the indicated resource service; s.d.=standard deviation. In view of the short study period of two 3-month intervals, mean resource use per patient was mostly once. 
Costs of inflammatory polyarthritis

For Review Only:

Supplementary material uploaded online for unit costs and expenditure questionnaires. 